\title{
Automatic daily remote monitoring in heart failure patients implanted with a cardiac resynchronisation therapy-defibrillator: a single-centre observational pilot study
}

Peter Ezer ${ }^{1}$, Nelli Farkas², István Szokodi ${ }^{1,3}$, Attila Kónyi ${ }^{1}$

${ }^{1}$ Heart Institute, University of Pécs, Medical School, Foreign Medical Sciences, Hungary
${ }^{2}$ Bioanalytical Institute, University of Pécs, Medical School, Pecs, Hungary
${ }^{3}$ Szentagothai Research Centre, University of Pécs, Pecs, Hungary

Submitted: 11 September 2020; Accepted: 26 December 2020

Online publication: 25 March 2021

Arch Med Sci 2023; 19 (1): 73-85

DOI: https://doi.org/10.5114/aoms/131958

Copyright ( 2021 Termedia \& Banach

\begin{abstract}
Introduction: The impact of remote monitoring (RM) on clinical outcomes in heart failure (HF) patients with cardiac resynchronisation therapy-defibrillator (CRT-D) implantation is controversial. This study sought to evaluate the performance of an RM follow-up protocol using modified criteria of the PARTNERS HF trial in comparison with a conventional follow-up scheme.

Material and methods: We compared cardiovascular (CV) mortality (primary endpoint) and hospitalisation events for decompensated HF, and the number of ambulatory in-office visits (secondary endpoint) in CRT-D implanted patients with automatic RM utilising daily transmissions (RM group, $n=45$ ) and conventional follow-up (CFU group, $n=43$ ) in a single-centre observational study.

Results: After a median follow-up of 25 months, a significant advantage was seen in the RM group in terms of CV mortality ( 1 vs. 6 death event, $p=0.04)$, although RM follow-up was not an independent predictor for CV mortality $(\mathrm{HR}=0.882 ; 95 \% \mathrm{Cl}: 0.25-3.09 ; p=0.845)$. Patient $\mathrm{CV}$ mortality was independently influenced by hospitalisation events for decompensated $\mathrm{HF}(\mathrm{HR}=3.24 ; 95 \% \mathrm{Cl}: 8-84 ; p=0.022)$ during follow-up. We observed significantly fewer hospitalisation events for decompensated HF (8 vs. 29 events, $p=0.046$ ) in the RM group. Furthermore, a decreased number of total (161 vs. 263, $p<0.01)$ and unnecessary ambulatory in-office visits (6 vs. $19, p=0.012$ ) were seen in the RM group as compared to the CFU group. Conclusions: Follow-up of CRT-D patients using automatic RM with daily transmissions based on modified PARTNERS HF criteria enabled more effective ambulatory interventions leading indirectly to improved CV survival. Moreover, RM directly decreased the number of HF hospitalizations and ambulatory follow-up burden compared to CRT-D patients with conventional follow-up.
\end{abstract}

Key words: survival, follow-up, heart failure, cardiac resynchronisation therapy, remote monitoring.

\section{Introduction}

Cardiac resynchronisation therapy (CRT) provides an evidence-level treatment manner in a well selected subgroup of patients with advanced systolic heart failure and functional dyssynchrony. Cardiac resynchronisa-

\author{
Corresponding author: \\ Kónyi Attila \\ Heart Institute \\ University of Pécs \\ Medical School \\ 13 Ifjusag St \\ 7602, Pécs, Hungary \\ E-mail: konyi.attila@pte.hu
}


tion therapy is a proven method to reduce symptoms, morbidity (hospitalisations), and mortality in heart failure patients responding or super-responding to therapy $[1,2]$. Remote monitoring of patients with cardiac implantable devices (CIED) in heart failure has an established recommendation according to the currently available heart failure guidelines of the European Society of Cardiology (ESC) [3]. Cardiac resynchronisation therapy-defibrillators (CRT-D), capable of remote monitoring (RM) function, transmit numerous measurable patient- and device-related data on a predetermined time basis or even immediately if a critical event is observed by the implanted device. Detection alerts and transmission algorithms depend on the manufacturer of the system.

It has been shown that RM is a safe and reliable method in the follow-up of patients with advanced heart failure and implantable cardiac defibrillators (ICD) [4-8]. The detection times of major adverse clinical events such as arrhythmia events, silent atrial fibrillation, inappropriate ventricular shocks, and even device-related malfunction and impending heart failure events are significantly shorter than with conventional in-office follow-up [7-10].

The PARTNERS HF study investigated 694 CRT defibrillator patients with remote monitoring and continuous multi-parameter monitoring for heart failure. Monthly review of monitored parameters and patients with positive combined heart failure diagnostics of long-lasting atrial fibrillation and/or high ventricular rate, low biventricular pacing ratio, abnormal autonomic signs (elevated resting heart rate, low heart rate variability), decreased patient activity, and high thoracic fluid index had a 5.5-fold increase in the risk for heart failure hospitalisations within the subsequent month. Evaluation of heart failure device diagnostics more frequent than 1 week improved the ability to risk stratify patients for subsequent heart failure events [11].

Although previous result are well proven, the exact alerting thresholds for each detection parameter are still debated, and a novel heart failure detection algorithm and effective intervention are highly warranted to prevent worsening heart failure-related hospitalisation and death.

Remote monitored heart failure patients implanted with cardiac implantable devices (CIEDS) show contradicting outcome results regarding survival, hospitalisation, and institutional ambulatory burden in prospective randomised studies and meta-analyses [12-16].

Nevertheless, several trials proved an equivocal decrease in institutional ambulatory burden and cost effectiveness in the care of remote monitored patients [17-22].

In this study, we tested our institutional RM heart failure detection algorithm protocol using adapted and refined PARTNERS HF criteria for an automated daily transmission enabling RM system (Biotronik Home Monitoring ${ }^{\mathrm{TM}}$ ). We assumed that refined RM detection criteria and early interventions aiming at prevention of decompensated heart failure events can decrease heart failure-related hospitalisations and increase survival compared to a conventional 'ambulatory-only'followed patient group, without increasing hospital ambulatory burden or the number of unscheduled in-office patient evaluations in an RM-followed patient group of CRT-D-implanted patients.

\section{Material and methods}

\section{Study design}

This investigation was a single-centre retrospective observational pilot study involving 2 parallel cohorts consisting of heart failure patients. All patients were implanted with Biotronik Iforia $^{\text {TM }}$ CRT-D devices from 2014 January to 2017 December in our university referral hospital. Patients received a de novo implanted CRT-D device in accordance with the current ESC guidelines for heart failure therapy [3]. All implanted CRT-D devices were eligible for remote monitoring. Cardiomessenger ${ }^{\mathrm{TM}}$ remote transmission devices were provided by the manufacturer, and the availability was not continuous during the implantation period. The opportunity for remote monitoring and device remote follow-up was offered to every patient before implantation if an RM eligible Iforia device and Cardiomessenger device were available at the same time. Remote transmission device availability was the main selection criterion, whether a patient was followed with remote monitoring or not.

Conventionally followed patients received an Iforia CRT-D device capable of RM function, as well. Only 1 of 44 patients in the conventionally followed (CFU) group refused remote monitoring follow-up; this patient was excluded from the study. The other 43 patients in the CFU group had no possibility to receive a remote transmission device at the time of implantation.

Patients were non-randomised is this study, but it should be noted that no significant differences were observed in the most important baseline characteristics between the 2 patient groups.

All patients signed a written informed consent form. All CRT-D devices and the automatic daily basis tele-monitoring system (Home Monitoring) were provided by Biotronik (Biotronik SE \& Co., KG, Berlin, Germany). Biotronik devices with a Home Monitoring remote monitoring system were chosen for the retrospective analysis because the system provides daily transmission based automatic remote monitoring, and the specific device was 
the most available in our institute at the time of device implantations.

Follow-up data of 88 de novo CRT-D-implanted patients were collected and analysed. The remote monitored CRT-D-implanted patients (RM group, $n=45$ ) were followed with automatic daily transmission-based continuous remote monitoring, and remote interrogation of the device was performed every 3 months. At least one scheduled yearly in-office follow-up visit was agreed with these patients. Alerts were received based on Home Monitoring's intrinsic alert algorithm. Remote transmissions, including alerts, were observed daily by a competent nurse staff, and all the relevant transmissions were immediately forwarded to a device/heart failure specialist.

CRT-D-implanted patients with conventional follow-up (CFU group, $n=43$ ) had a scheduled in-clinic ambulatory appointment every 3-6 months during follow-up, depending on the treating cardiologist/ device specialist. Table I presents the parameters that were assessed at remote interrogation and/or in-office ambulatory follow-up events.

Data regarding CV mortality, cardiovascular hospitalisations, institutional admissions for decompensated heart failure, ambulatory patient flow, baseline characteristics, medications, and comorbidities were collected from patient files, remote interrogations of the device, and from an integrated patient care information system of University of Pécs. Data collection was performed in accordance with international regulations regarding the protection of personal information and data. All subjects gave their informed consent for inclusion before they participated in the study. The study was conducted in accordance with the Declaration of Helsinki, and the protocol was approved by the Ethics Committee of the University of Pécs (6600/2020).

\section{Study endpoints}

The primary objective of this study was to compare the CV mortality of remote-monitored patients with patients on a conventional follow-up scheme. Survival was assessed as the time from CRT-D implantation to a CV mortality event. Secondary endpoints were the number of cardiovascular hospitalisations, expressively the number of hospitalisations for decompensated heart failure. Further secondary endpoints were the total number of ambulatory visits, and the ratio of unnecessary ambulatory visits in each patient group during follow-up.

\section{Novel detection algorithm for worsening heart failure in the remote monitoring group}

Several parameters served as additional accessible information for the heart failure status of the patient in the RM group during follow-up. The PARTNERS HF prospective multi-centre observational study [11] published a combined heart failure device algorithm for predicting an upcoming heart failure event. The algorithm consisted of long atrial fibrillation duration ( $>6 \mathrm{~h} /$ day for at least 1 day, without persistent AF), rapid ventricular rate (daily average above 90/min for at least 7 days), high thoracic fluid index (above $60 \mathrm{Ohms}$ ), low patient activity below $1 \mathrm{~h} /$ day over 7 days, high nocturnal ventricular rate (> 85/min for 7 consecutive days), low heart rate variability (< $60 \mathrm{~ms}$ for 7 days), low biventricular pacing ratio (<90\% for 5 of 7 days), or at least 1 ventricular shock event. The algorithm was considered positive if a patient had 2 positive criteria during a 1-month period. Vamos et al. [23] investigated thoracic fluid index alerts in a prospective observational study and refined the PARTNERS HF algorithm to a modified version, increasing the algorithms specificity to $86.5 \%$ and sensitivity to $93.8 \%$ in predicting an upcoming heat failure event [24]. In our study, the automated daily continuous remote monitoring method allowed assessment of patient activity level, ventricular heart rate at rest, heart rate variability, intrathoracic impedance tendency, biventricular pacing ratio, and all

Table I. Parameters assessed at remote interrogation and in-office follow-up

\begin{tabular}{|c|c|}
\hline Parameter type & \\
\hline Current rhythm diagnosis and pacemaker dependency & $\begin{array}{l}\text { Therapy given for sustained ventricular arrhythmia } \\
\text { (anti-tachycardia pacing, ventricular shock)* }\end{array}$ \\
\hline Mean ventricular heart rate* & Biventricular pacing ratio* \\
\hline Battery lifetime expectancy & Inappropriate ventricular shock events* \\
\hline Lead impedance/shock lead impedances & Review of device-triggered alert events* \\
\hline Pacing thresholds for different electrodes & Patient activity level ${ }^{*}$ \\
\hline Sensing signal amplitude threshold for different electrodes & Heart rate variability* \\
\hline $\begin{array}{l}\text { All arrhythmia events (atrial arrhythmia burden, ventricular } \\
\text { extrasystoles, and other arrhythmia events) }{ }^{\star}\end{array}$ & Intrathoracic impedance status* \\
\hline
\end{tabular}


arrhythmia and anti-tachycardia therapy events. In the CFU group, these data were only available every 3-6 months at in-office follow-ups. Table II compares refined PARTNERS HF criteria and our institutional remote monitoring criteria for an upcoming decompensated heart failure event.

In our criteria system the major predictors for a heart failure event were sustained ventricular arrhythmia, ventricular appropriate or even inappropriate shock or anti-tachycardia pacing events, and new-onset atrial fibrillation burden exceeding $6 \mathrm{~h}$ for at least one day. Upon detection of even 1 major criterion, patients were called in for an unscheduled visit. Minor criteria were a decrease of thoracic impedance of at least $20 \%$ in the last 7 days, a decrease of biventricular pacing ratio below $90 \%$ in the last 7 days, a marked decrease $(<1 \mathrm{~h}$ a day) of patient activity level in the last 7 days, a marked decrease in heart rate variability ( $<60 \mathrm{~ms}$ ) in a week, or an increased resting ventricular heart rate for 7 days (>90/min). If no major but at least 2 minor RM criteria for worsening heart failure state were positive at remote interrogation, an immediate direct telephone consultation was made with the patient and even minor symptoms associated with an impending cardio-circulatory decompensation were interrogated. If the patient's symptoms were positive, an unscheduled urgent in-office visit was arranged. Unscheduled ambulatory visits had the aim for a pre-emptive medical- or device-mediated intervention, thus preventing patients from further deterioration and hospitalisation for decompensated heart failure. Our institutional criteria-protocol for screening remote monitored patients with impeding status for decompensated heart failure is shown in Figure 1.

\section{Ambulatory visit definitions}

Patient flow at our institute was assessed with respect to all ambulatory visits in both patient groups, divided into scheduled, unscheduled necessary, and unscheduled unnecessary ambulatory visit events. Scheduled ambulatory visits were always in the form of a prearranged ambulatory appointment at least once a year in the RM group and every 3 to 6 months in the CFU group. Unscheduled ambulatory in-office visits only occurred in the RM group if at least 1 major RM criteria for heart failure or at least 2 minor criteria with even modest heart failure symptoms at patient interrogation occurred. These patients were urgently contacted, and a pre-emptive unscheduled ambulatory visit was arranged. Patients in the CFU group were checked at scheduled visits; unscheduled visits were only set up based on general physician referral, emergency physician referral, or severe patient complaints. Unscheduled ambulatory visits qualified as unnecessary inoffice visits were visit events where no CRT-D device programme modifications, no new cardiovascular drug administration or dose modification, and no subsequent therapy or cardiovascular hospitalisation were performed (Figure 1).

\section{Statistical analysis}

All follow-up variables were divided to categorical or continuous variables. Data are presented as mean \pm standard deviation for normally dis-

Table II. Comparison of refined PARTNERS HF [22] and remote monitoring criteria for predicting decompensated heart failure events in our institute

\begin{tabular}{|c|c|c|}
\hline Device parameter & Refined PARTNERS HF criteria [22] & $\begin{array}{l}\text { Remote monitoring criteria for } \\
\text { decompensated heart failure event }\end{array}$ \\
\hline Thoracic fluid index alert & Elevated thoracic fluid index (> $60 \mathrm{Ohm}$ ) & $\begin{array}{c}>20 \% \text { decrease in thoracic impedance } \\
\text { value for } 7 \text { days }\end{array}$ \\
\hline New onset AF episode & $\begin{array}{c}A F>6 \text { h on at least one day } \\
\text { without persistent } A F\end{array}$ & $\begin{array}{c}\text { New onset } A F \text { at least } 6 \mathrm{~h} \text { a day } \\
\text { without persistent } A F\end{array}$ \\
\hline Ventricular rate during AF & $\begin{array}{c}A F>24 \mathrm{~h} \text { and daily average ventricular } \\
\text { rate during } A F>90 / \mathrm{min}\end{array}$ & Not used \\
\hline $\begin{array}{l}\text { Average daily ventricular } \\
\text { heart rate }\end{array}$ & Not used & $>90 /$ min for 7 consecutive days \\
\hline Patient activity level & Lower average activity in the past 5 days & Lower average activity in the past 7 days \\
\hline Nocturnal heart rate & $\begin{array}{l}\text { Average night rate }>85 / \mathrm{min} \text {, or elevated } \\
\text { with } 20 \text { over the past } 5 \text { days }\end{array}$ & Not used \\
\hline Heart rate variability & $<60$ ms every day for 1 week & $<60$ ms every day for 1 week \\
\hline Biventricular pacing ratio & $<90 \%$ in the past 5 days & $<90 \%$ in the past 7 days \\
\hline Ventricular arrhythmias & $\begin{array}{l}\text { Ventricular shock or anti-tachycardia } \\
\text { pacing events }\end{array}$ & $\begin{array}{l}\text { Ventricular shock, anti-tachycardia } \\
\text { pacing events, or sustained ventricular } \\
\text { arrhythmias without therapy }\end{array}$ \\
\hline
\end{tabular}


tributed continuous variables, median $\left(25^{\text {th }}\right.$ and $75^{\text {th }}$ percentiles) for non-normally distributed variables, or percentages for binary variables. Missing data were not replaced; all available data were used for sample distribution evaluation. Normality was checked with the Kolmogorov-Smirnov test. For normally distributed data Student's $t$-test was used. The Mann-Whitney test was used for inter-individual comparisons of continuous variables when normality was rejected. Categorical variables were compared with the $\chi^{2}$ or Fisher's exact test. For cardiovascular survival analysis we applied Kaplan-Meier survival curve estimation with log rank test and Cox's regression with forward selection. Statistical analysis was performed using IBM SPSS statistical software version 25.0. (Armonk, NY, IBM Corp.). Post hoc power analysis was performed for the primary endpoint outcome (cardiovascular mortality) based on Kaplan-Meier survival analysis using Stata version 15 (StataCorp. 2017. Stata Statistical Software: Release 15. College, TX: StataCorp LLC.). The level of significancy was defined as $p<0.05$.

\section{Results}

\section{Patient populations}

Total of 88 CRT-D recipients were included in the study. Patient baseline characteristics are summarised in Table III. Despite patients being non-randomised in this study, RM and CFU patient groups did not differ significantly in most baseline features. There were no significant differences regarding patient age (59.7 vs. 69.6; $p=0.2$ ), female gender (12 vs. $7 ; p=0.23$ ), baseline left ventricular ejection fraction (29.49 vs. 30.27; $p=0.47)$, New York Heart Association (NYHA) functional class (2.82 vs. 2.88; $p=0.202$ ), or number of patients with left bundle branch block (LBBB) morphology (42 vs. $40 ; p=0.95$ ) at the time of device implantation. The 2 patient populations showed no significant differences with respect to anamnestic cardiovascular comorbidities, number of ischaemic cardiomyopathies ( 25 vs. $25 ; p=0.86$ ), paroxysmal or permanent atrial fibrillations (11 vs. $11 ; p=0.9)$, chronic obstructive pulmonary disease ( 9 vs. $9 ; p=0.499$ ), chronic kidney disease (CKD) stage 3 defined as glomerular filtration ratio (GFR) between $60 \mathrm{ml} / \mathrm{min}$ and $30 \mathrm{ml} / \mathrm{min}$ (2 vs. 3; $p=0.673$ ). Patients with a GFR below $30 \mathrm{ml} / \mathrm{min}$ were not included in this study. No significant differences were seen at baseline cardiovascular medical regime except for higher statin usage in the RM group ( 23 vs. $13 ; p=0.008$ ), no difference in point of baseline heart failure medication like ACE inhibitor/angiotensin receptor inhibitor (37 vs. 37; $p=0.59$ ), $\beta$ receptor blocker (41 vs. 39; $p=1.0)$, mineralocorticoid receptor antagonist (31 vs. $24 ; p=0.31$ ), diuretics (40 vs. $39 ; p=1.0$ ), and amiodarone (14 vs. $9 ; p=0.377$ ) usage. Anticoagulant (20 vs. $26 ; p=0.12$ ) and antiplatelet agent usage (22 vs. 20; $p=0.991$ ) were also comparable at baseline in the RM and CFU groups.

\section{Improved cardiovascular survival and less hospitalisation for heart failure in the remote monitoring group}

Significantly lower CV mortality was observed (1 vs. $6 ; p=0.04$ ) in the RM group during follow-up (Figure 2). The Kaplan-Meier estimate of 1-year CV mortality was $1.45 \%$ in the RM group and $6.92 \%$

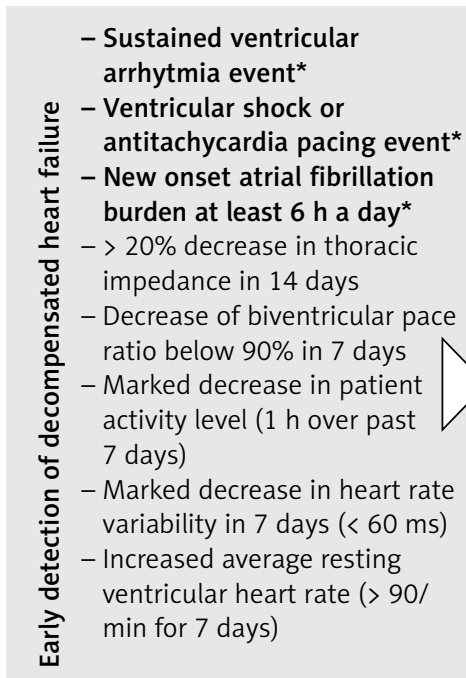

At least 2 predictors present OR 1 major predictor*
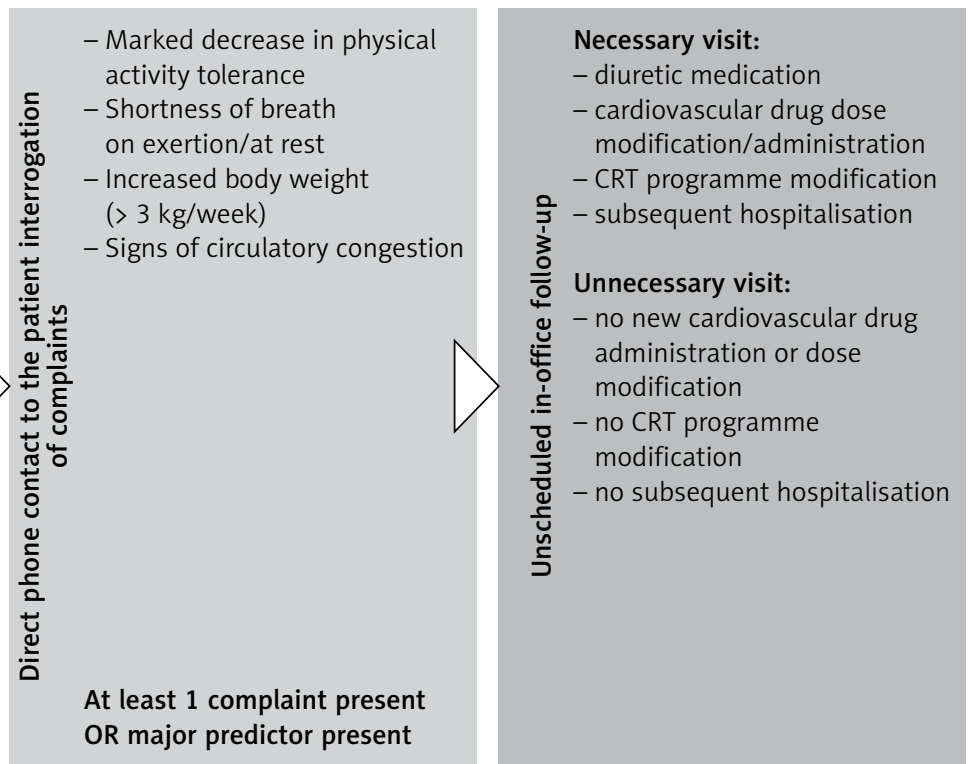

Figure 1. Institutional remote monitoring protocol for early detection of decompensated heart failure in cardiac resynchronisation therapy-defibrillator-implanted heart failure patients. Major predictors for impeding heart failure event were marked as *. At least two minor criteria positivity resulted in direct patient contact 
Table III. Comparison of baseline patient characteristics

\begin{tabular}{|c|c|c|c|}
\hline Characteristic & RM group $(n=45)$ & CFU group $(n=43)$ & $P$-value \\
\hline Age [years], mean (SD) & $59.7(10.6)$ & $62.6(10.5)$ & 0.200 \\
\hline Female, $n(\%)$ & $12(26.7)$ & $7(16.3)$ & 0.230 \\
\hline $\begin{array}{l}\text { Left ventricular ejection fraction (\%), } \\
\text { mean (SD) }\end{array}$ & $29.49(5.1)$ & $30.27(4.4)$ & 0.471 \\
\hline NYHA class, mean (SD): & $2.82(0.71)$ & $2.88(1.41)$ & 0.202 \\
\hline II, $n(\%)$ & $15(33.3)$ & $9(20.9)$ & \\
\hline III, $n(\%)$ & $23(51.1)$ & $30(69.8)$ & \\
\hline IV, $n(\%)$ & $7(15.6)$ & $4(9.3)$ & \\
\hline Left bundle branch block, $n(\%)$ & $42(93.3)$ & $40(93.0)$ & 0.950 \\
\hline Ischemic aetiology, $n$ (\%) & $25(55.5)$ & $25(58.1)$ & 0.860 \\
\hline Hypertension, $n$ (\%) & $35(77.8)$ & $35(81.4)$ & 0.674 \\
\hline Diabetes, $n(\%)$ & $17(37.8)$ & $13(30.2)$ & 0.821 \\
\hline Hyperlipidaemia, $n$ (\%) & $11(24.4)$ & $8(18.6)$ & 0.543 \\
\hline COPD, $n(\%)$ & $6(13.3)$ & $8(18.6)$ & 0.499 \\
\hline Chronic kidney disease, $n$ (\%) & $2(4.4)$ & $3(6.9)$ & 0.673 \\
\hline Atrial fibrillation, $n(\%)$ & $11(24.4)$ & $11(25.6)$ & 0.900 \\
\hline \multicolumn{4}{|l|}{$\begin{array}{l}\text { Medications at the time } \\
\text { of implantation: }\end{array}$} \\
\hline$\beta$-receptor blockers, $n$ (\%) & $41(91.1)$ & $39(90.7)$ & 1.000 \\
\hline $\mathrm{ACEi} / \mathrm{ARB}, n(\%)$ & $37(82.2)$ & $37(86.0)$ & 0.590 \\
\hline MRA, $n(\%)$ & $31(68.9)$ & $24(55.8)$ & 0.310 \\
\hline Diuretics, $n(\%)$ & $40(88.9)$ & $39(90.7)$ & 1.000 \\
\hline Amiodarone, $n(\%)$ & $14(31.1)$ & $9(20.9)$ & 0.377 \\
\hline Anticoagulants, $n(\%)$ & $20(44.4)$ & $26(60.5)$ & 0.120 \\
\hline Antiplatelet agent, $n$ (\%) & $22(48.9)$ & $20(46.5)$ & 0.991 \\
\hline Statin, $n(\%)$ & $27(60.0)$ & $13(30.2)$ & 0.008 \\
\hline
\end{tabular}

NYHA class - New York Heart Association class, COPD - chronic obstructive pulmonary disease, ACEi - angiotensin-converting-enzyme inhibitor, ARB - angiotensin-receptor blocker, MRA - mineralocorticoid-receptor-antagonist.

in the CFU group. Notably, post hoc power analysis with a 2 -sided $\alpha$ of $5 \%$ and a statistical power of $88 \%$ was obtained for CV survival outcome.

Potential parameters for predicting CV mortality were divided into 3 parameter subgroups. Relevant patient baseline characteristics, follow-up parameters, and medication factors were analysed for predicting CV mortality in our patient cohort. Cox-regression analysis showed that, among baseline characteristics, NYHA class $(\mathrm{HR}=2.69 ; 95 \% \mathrm{Cl}: 0.01-7.17 ; p=0.047)$ was an independent predictor. Among follow-up factors, only the occurrence of a hospitalisation event for decompensated heart failure $(\mathrm{HR}=3.24 ; 95 \% \mathrm{Cl}$ : $1.19-8.84 ; p=0.022$ ) was a significant, independent predictor for CV mortality. Other clinically relevant factors like female sex, ischaemic heart failure aetiology, baseline left ventricular ejection fraction, relevant cardiovascular comorbidities, ventricular shock events, unscheduled ambulatory visit events, baseline medical regime, or even remote monitoring follow-up method were not independent predictors of CV mortality in our patient cohorts (Figure 3).

Although cardiovascular hospitalisations (37 vs. $46 ; p=0.076)$ or the number of in-hospital spent days did not differ significantly (245 vs. 346; $p=0.35$ ), in terms of hospitalisation events for decompensated heart failure we noted a significant difference, with the RM group performing better (8 vs. $29 ; p=0.046$ )

Echocardiographic control for evaluation of left ventricular function was reassessed 6-12 months after device implantation. No differences were 
seen in control of left ventricular ejection fraction between the 2 observed groups (33.1 vs. 32.2; $p=0.91)$ (Table IV).

\section{Hospitalisation, arrhythmias, and defibrillator therapy}

Institutional admissions for novel or high-ventricular rate atrial fibrillation treatment, cumulative ventricular arrhythmias/ventricular shocks, or general check-up prior to heart transplantation were registered in both groups. We noted a trend for higher count in the RM group in atrioventricular node ablation procedures and other device-related operative procedures: 4 pacing electrode change/repositioning and 4 pocket haematoma evacuations were performed in the RM group, whereas 2 pacing electrode change/repositioning and 1 pocket haematoma evacuation in the CFU group were performed (Figure 4).

Comparable results were seen between the 2 groups regarding incidence of ventricular arrhythmias ( 243 vs. $205 ; p=0.067$ ) or ICD therapeutic response to arrhythmia (anti-tachycardia pacing (114 vs. $81 ; p=0.876)$, appropriate ventricular shocks (100 vs. $88 ; p=0.23)$ ) respectively; even the count of inappropriate ventricular shocks (11 vs. $13 ; p=0.83$ ) or patient number affected by inappropriate shocks ( 4 vs. $3 ; p=0.74$ ) did not differ markedly (Table IV).

\section{Effectivity of institutional ambulatory care}

During median 30 months of follow-up 38,521 daily remote transmissions were made, and 93\% of remote transmissions were successful in the RM group. Detection algorithm positivity for major/minor predictors of an upcoming decompensated heart failure event were assessed weekly with the help of competent nursing staff and an onsite device/heart failure specialist.

Significant results were seen in connection with ambulatory patient flow. During a 2-year follow-up period, there were significantly fewer (as much as 39\% lower) total ambulatory in-office visits (161 vs. $263 ; p<0.01$ ) in the RM group as compared to the CFU group. A numerically higher number was observable with respect to unscheduled ambulatory visit events in RM group (36 vs. 22; $p=0.167$ ), but this difference was not statistically significant. The number of unscheduled unnecessary ambulatory visits was significantly lower in the RM group ( 6 vs. $19 ; p=0.012$ ). Figure 5 shows the improved efficacy of ambulatory patient flow in the remote monitoring group.

In the RM arm, of those 30 unscheduled necessary ambulatory visits, 27 in-office patient evaluations were arranged because of worsening heart failure alert positivity seen during remote trans-

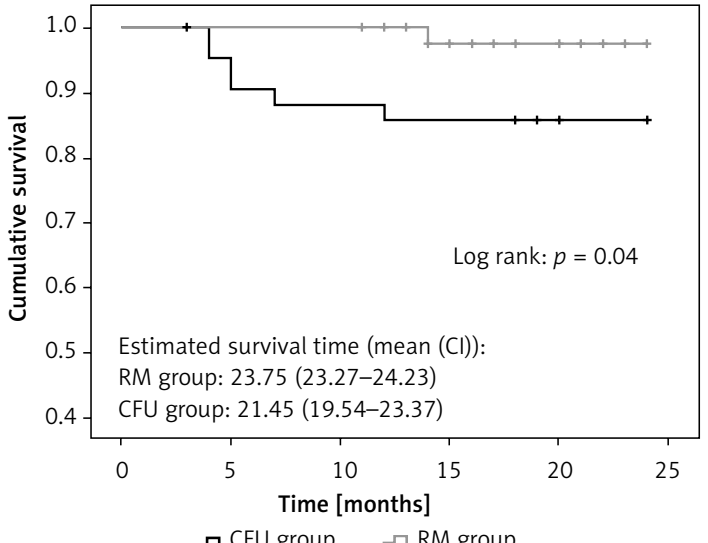

Pts. at risk

$\begin{array}{lllllll}\text { RM } & 45 & 45 & 45 & 39 & 35 & 31 \\ \text { CFU } & 43 & 37 & 36 & 35 & 33 & 32\end{array}$

Figure 2. Kaplan-Meier estimation of cardiovascular mortality. Significantly better cardiovascular survival ( 1 vs. 6 cases; $p=0.04$ ) was observed in the remote monitoring patient group after 25 months of investigation

RM group - remote monitoring group, CFU group conventional follow-up group

missions using our institutional algorithm. Twenty of 27 patients required higher diuretic dose because of circulatory congestion and/or meaningful decrease in thoracic impedance value, but only 8 patients required hospitalisation for decompensated heart failure, severe dyspnoea, cardio-respiratory failure, or severe congestion signs.

Eighteen patients required further cardiovascular medication modification, and only 4 required significant CRT device program modifications in ambulatory settings.

Six patients had novel atrial fibrillation burden exceeding $6 \mathrm{~h}$ with rapid ventricular rate and low biventricular pacing ratio. All the 6 patients required hospitalisation, and 3 patients required further hospitalisation for atrioventricular node ablation procedure as a consequence of medically refractory high ventricular rate atrial fibrillation. All patients required hospitalisation with major ventricular sustained arrhythmia and $>1$ inappropriate/appropriate ventricular shocks.

\section{Discussion}

This study sought to evaluate the impact of a novel remote monitoring heart failure detection algorithm, designed for an automated daily transmission-based remote monitoring system.

Our RM detection algorithm seems to show efficacy at improving advanced heart failure patient survival, decreasing heart failure-related hospitalisations and institutional ambulatory patient burden beneath more effective in-office patient care even in our pilot study with moderate patient cohorts. 
A

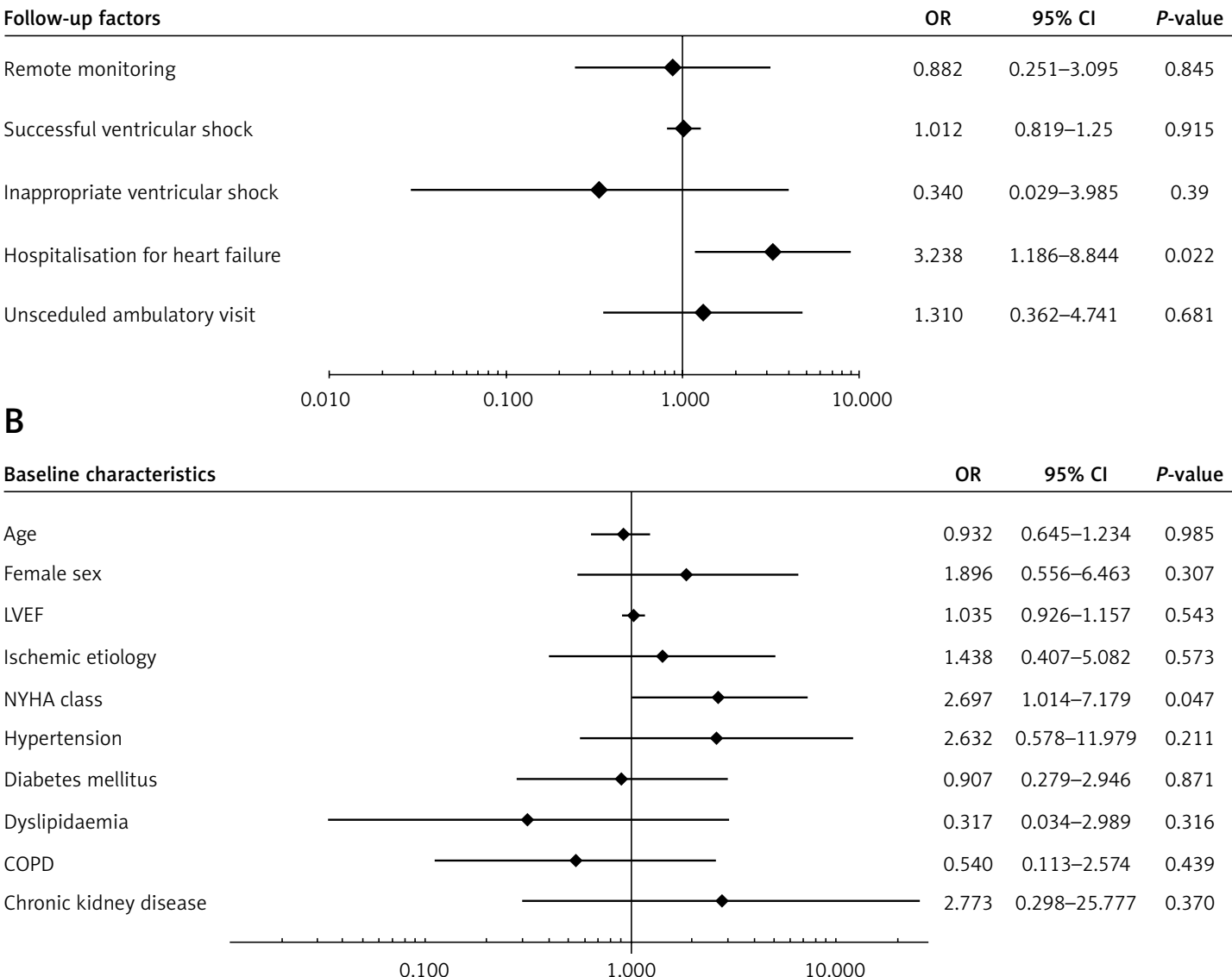

C

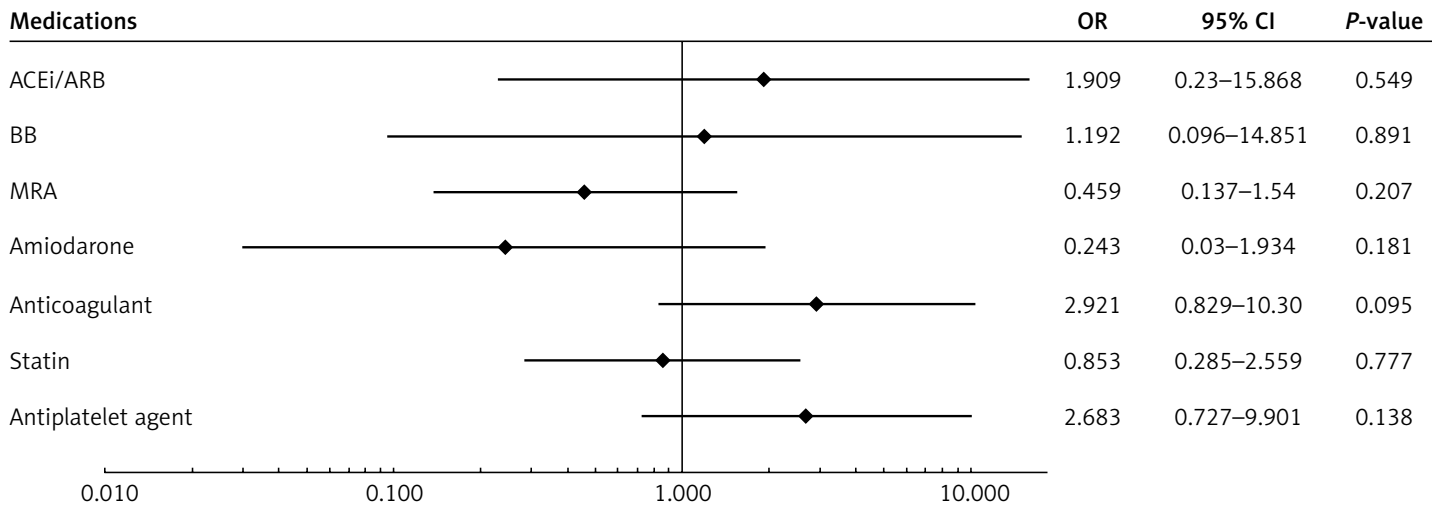

Figure 3. Variable regression analysis for predictors of mortality in 3 parameter groups (follow-up parameters (A), baseline characteristics (B), medications (C)). Analysis for cardiovascular mortality predictors was performed in 3 different parameter groups (A, B, C). New York Heart Association class $(p=0.047)$ and hospitalisation event for decompensated heart failure $(p=0.022)$ were significant predictors of cardiovascular mortality in our patient cohorts LVEF - left ventricular ejection fraction, NYHA - New York Heart Association, COPD - chronic obstructive pulmonary disease, $A C E i$ - angiotensin-converting-enzyme inhibitor, $A R B$ - angiotensin-receptor-antagonist, $B B$ - $\beta$-receptor blocker, MRA - mineralocorticoid-receptor-antagonist.

The daily data transmission-based algorithm seems important in improving patient outcomes, because tele-monitoring algorithms, typically with weekly data transmission (MORE CARE study), failed to prove the benefit of remote monitoring in heart failure patients [14].
Implant-based automatic daily multi-parameter tele-monitoring of CIED patients with heart failure (IN-TIME study) first showed survival benefit in the remote monitoring arm in a patient group consisting of dual-chamber ICD- or CRT-D-implanted patients. The RM system transmitted data on 
Table IV. Follow-up-related results

\begin{tabular}{|c|c|c|c|}
\hline Characteristic & RM group $(n=45)$ & CFU group $(n=43)$ & $P$-value \\
\hline Follow-up time [months], median (IQR) & $30(20-39)$ & $24(16-33)$ & 0.06 \\
\hline Cardiovascular mortality, $n$ (\%) & $1(2.2)$ & $6(13.9)$ & 0.04 \\
\hline Cardiovascular hospitalisation events, $n$ & 37 & 46 & 0.76 \\
\hline Days spent for cardiovascular hospitalisations, $n$ & 245 & 346 & 0.35 \\
\hline $\begin{array}{l}\text { Hospitalisation events for decompensated heart } \\
\text { failure, } n\end{array}$ & 8 & 29 & 0.046 \\
\hline Total ambulatory visits, $n$ & 161 & 263 & $<0.01$ \\
\hline Unscheduled ambulatory visits, $n$ & 36 & 22 & 0.167 \\
\hline Unscheduled unnecessary ambulatory visits, $n$ & 6 & 19 & 0.012 \\
\hline Sustained ventricular arrhythmias, $n$ & 243 & 205 & 0.067 \\
\hline Anti-tachycardia pacing events, $n$ & 114 & 81 & 0.876 \\
\hline Appropriate, successful ventricular shocks, $n$ & 100 & 88 & 0.23 \\
\hline Inappropriate ventricular shocks, $n$ & 11 & 13 & 0.83 \\
\hline Patients with inappropriate ventricular shocks, $n(\%)$ & $4(8.8 \%)$ & $3(6.9 \%)$ & 0.74 \\
\hline Biventricular pace ratio (\%), mean ( \pm SD) & $98.9(8.0)$ & $98.7(6.6)$ & 0.93 \\
\hline $\begin{array}{l}\text { Control left ventricular ejection fraction (\%), } \\
\text { mean }( \pm S D)\end{array}$ & $33.1(9.69)$ & $32.2(11.1)$ & 0.91 \\
\hline
\end{tabular}

daily basis; thus, the opportunity for closer heart failure status monitoring and management was given as compared to conventional care. The RM was associated with a $60 \%$ relative decrease in 1-year CV mortality in the IN-TIME trial; however, the RM group and the control group did not differ significantly for the number of hospital admissions for worsening heart failure [13].

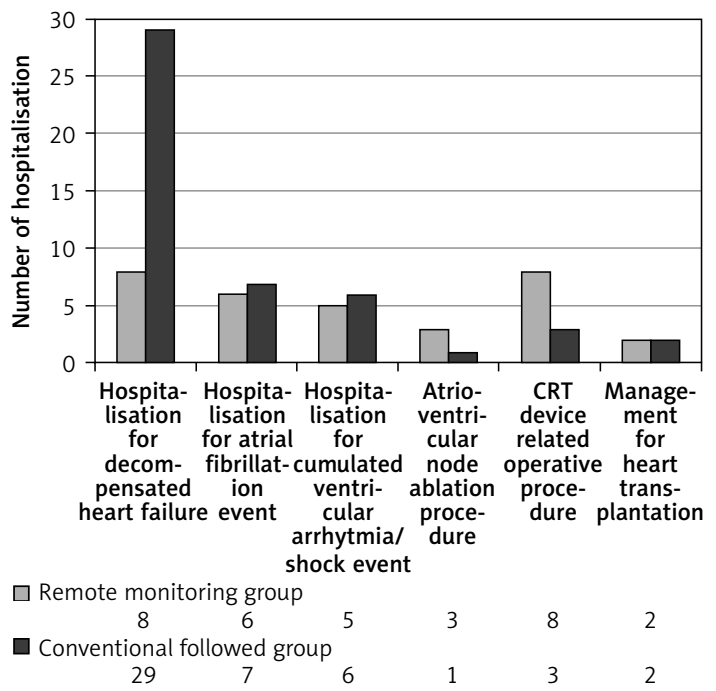

Figure 4. Distribution of cardiovascular hospitalisation events during follow-up. Higher number of hospitalisations for worsening heart failure is observable in the CFU group ( 8 vs. $29, p=0.046$ ). There were no significant differences regarding other cardiac or device-related hospitalisation events
In a recent meta-analysis by Klersy et al., RM follow-up failed to show a decrease in the total number of cardiovascular hospitalisations, but RM was associated with a reduction in total ambulatory visit count [15]. In another meta-analysis, Parthiban et al. demonstrated comparable allcause mortality, cardiovascular mortality, and hospitalisation outcomes in ICD patients with RM or

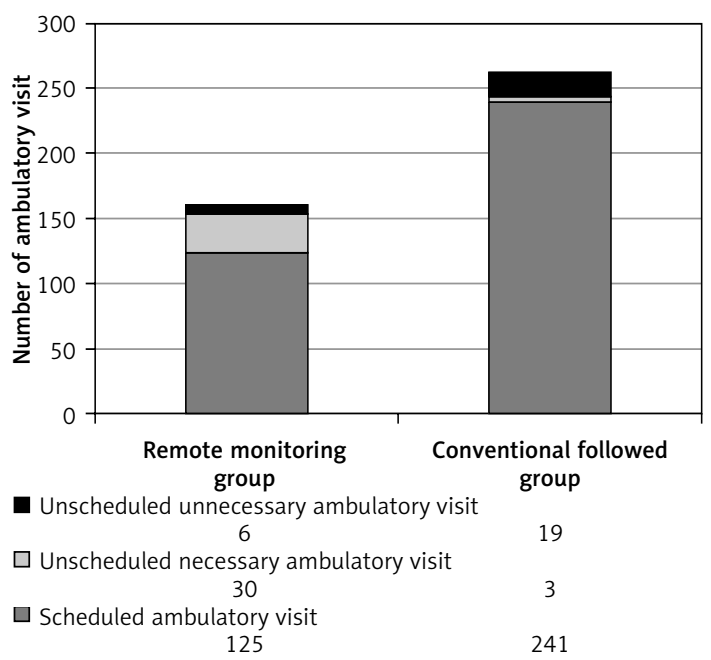

Figure 5. Ambulatory follow-up burden in the $2 \mathrm{pa}$ tient cohorts. Ambulatory patient flow graph shows a total $39 \%$ (161 vs. 263) reduction of ambulatory admissions between the 2 patient groups $(p<0.01)$. A significantly lower (6 vs. $19 ; p=0.012$ ) number of unnecessary ambulatory patient admissions was also observed 
conventional follow-up. However, a decrease in allcause mortality was observed in those trials using RM systems with daily data transmission [16].

The latest meta-analysis consisting of 3 large trials (TRUST, ECOST, IN-TIME) all with automated daily transmission-based remote monitoring in heart failure CIED patients showed a reduced composite endpoint of worsening heart failure hospitalisations and cardiovascular death; however, unscheduled in-office visit numbers were not lower in the RM-followed group [25]. None of the above-mentioned trials and meta-analyses reported improved cardiovascular patient survival, decreased hospitalisation rate for heart failure, or decreased institutional ambulatory burden with improved efficacy of the ambulatory care in the same remote-monitored advanced heart failure CIED patient population.

Nowadays, in spite of advanced multi-parameter RM techniques, evidence is still lacking regarding optimisation of early detection and fast intervention of CIED patients with higher risk of an impeding heart failure event, but previous literature has applicable data on several monitored parameters. Modern devices capable of measuring the patient's intrathoracic fluid status accelerate the early detection of patients with impending decompensated heart failure status. Thoracic impedance value change is the most widely studied factor, but evidence is lacking regarding optimal intervention thresholds for different device manufacturers. Intrathoracic fluid status is measured continuously by the implanted device in the form of intrathoracic impedance. As intrathoracic fluid accumulates, the intrathoracic impedance value decreases [25-29]. Although the predictive value of this parameter is well established in the literature, a single heart failure parameter alone seems to be too weak in daily practice to enable early and effective clinical intervention. PARTNERS HF [11] and modified PARTNERS HF criteria [23] both use a multi-parameter monitoring algorithm with monthly review to define patients with higher risk for heart failure decompensation. In these studies, a decrease in thoracic impedance value defines a higher risk patient group for an upcoming heart failure event. Furthermore, additional lower patient activity level, increased nocturnal ventricular heart rate, and suboptimal biventricular pacing ratio seemed to be the best independent predictors for heart failure events in patients with elevated intrathoracic fluid status [23]. We adapted modified PARTNERS HF criteria to Biotronik CRT defibrillators capable of daily remote transmission and refined the prediction criteria based on well-documented previous literature and clinical experience.

Major predictors were sustained ventricular arrhythmia and ventricular shock events. More than one ventricular arrhythmia and/or ventricular shock event independently and strongly affected patient survival of advanced systolic heart failure patients with ICD; thus, urgent unscheduled in-office patient evaluation seems substantial in this clinical situation [30, 31]. New-onset atrial fibrillation burden exceeding $6 \mathrm{~h}$ and higher ventricular rate are often the cause of lower biventricular pace ratio, functional worsening, and deterioration of heart failure status in CRT implanted patients [13].

Minor detection criteria prediction thresholds were optimised to have adequate sensitivity for the detection of impeding decompensation events. In the case of at least 2 minor criteria positivity and fast consultation and interrogation of even minor patient complaints seemed to improve the ability for pre-emptive adequate HF therapy in these patients. Minor criteria like elevated resting ventricular heart rate and sudden decrease in heart rate variability are important markers of autonomic response in advanced heart failure, and both parameters correlate with worse clinical outcomes, increased count of heart failure events, and cardiovascular death [32-34]. According to current guidelines for cardiac resynchronisation therapy, the biventricular pacing ratio of patients should be optimally as close to $100 \%$ as possible. Markedly decreased biventricular pacing ratio (e.g. $<80 \%$ for $48 \mathrm{~h}$ in IN-TIME study) was one of the main findings for an upcoming heart failure event in different trials [13, 23, 35].

It should be noted that besides well-defined parameter thresholds in the case of decreased patient activity, heart rate variability, and increased resting ventricular heart rate, it is almost impossible to define the exact intervention threshold, and a patient-individualised clinical decision should be proposed.

In our RM cohort, the remote monitoring followup method was not an independent predictor for patient cardiovascular mortality in our investigation; however can be assumed that the lower count of hospitalisation for decompensated heart failure observed in the RM group may directly and independently play an important role in lower cardiovascular mortality compared to conventionally followed patients.

Several national and international studies have dealt with the cost-effectiveness of remote monitoring. These studies revealed a significant reduction in costs for the health care system, primarily via the reduction in the costs of institutional ambulatory burden and in-office care services [18-22, 36]. As seen in the MORE CARE study [14], during a median 25-month follow-up, a clearly significant $41 \%$ decrease in expected ambulatory patient flow was observable at the health care institution, and the RM group had a significantly higher num- 
ber of unscheduled ambulatory in-office patient evaluations. In spite of the above findings, with the use of our remote monitoring detection algorithm a significant (nearly $39 \%$ ) reduction in total ambulatory flow in the RM group was observed, and there was a numerically higher but not significantly increased number of unscheduled visits in the RM group (36 vs. 22; $p=0.167$ ), but unscheduled in-office visits had a higher ratio of clinically necessary patient evaluations (30 vs. 3 events), mainly driven by pre-emptive medical and device-related ambulatory interventions preventing patients from further worsening of heart failure status and subsequent hospitalisation. These findings suggest that unscheduled unnecessary visits have been minimised during RM follow-up, and it is mostly due to higher sensitivity for clinically relevant events. These results let us conclude that there is an increased effectiveness of institutional ambulatory care in this patient group. Furthermore, this novel RM-based follow-up algorithm seems to have the ability to replace most routine ambulatory visits that would not require any intervention.

We should note that RM requires good patient adherence to follow-up and therapy. Improving adherence in this patient group is important to improve clinical outcomes and quality of life [37].

COVID-19 (corona virus disease 2019) accelerated the need for eligible heart failure patient monitoring systems for patients with or without a cardiac implanted device. Achieving appropriate social distancing during lockdowns but still the possibility for close heart failure monitoring became essential for these patients. Tele-monitoring and "virtual visit" events have gained in importance in the last months of the pandemic. Several HF management-guiding principles have been recommended from experts in the field recently $[38,39]$. Remote monitoring-mediated follow-up became more prominent in the last few months, and it will potentially play a valuable role in the follow-up of advanced heart failure CIED patients in the near future.

There are some limitations to address in our pilot study. Our 2 patient cohorts were selected retrospectively from our single university institute centre in Hungary. Patient cohorts consisted of 45 vs. 43 patients, and further patient enrolment to increase the sample size and statistical power was limited. However, it should be emphasised that post hoc power analysis of the primary outcome revealed $88 \%$ power and for secondary endpoint outcome $-99 \%$ with a $5 \%$ value of $\alpha$. This investigation was a non-randomised observational study. Remote transmission device availability and patient's decision for remote monitoring follow-up should be taken into consideration when we assess outcomes. Allocation to the RM follow-up arm of the study could improve patient adherence to medication and health improvement targets. These factors might improve outcomes in the RM group, although the 2 selected patient groups did not differ significantly in the most important clinical baseline features.

In conclusion, a novel heart failure detection algorithm based on modified PARTNERS HF criteria adapted to automated, daily data transmission-based remote monitoring-mediated follow-up, early patient contact, and intervention before an impeding heart failure event seemed to be associated with a lower number of heart failure hospitalisations as well as decreased institutional in-office follow-up burden and more efficient ambulatory care.

In our pilot study, remote monitoring-mediated follow-up played a role in the improvement of cardiovascular mortality outcomes compared to conventionally followed CRT-D patients. Further randomised trials with major patient populations are needed to confirm the results observed in our study.

\section{Acknowledgments}

This research was funded by the National Research, Development, and Innovation Office of Hungary (NKFIH K120536).

\section{Conflict of interest}

The authors declare no conflict of interest.

\section{References}

1. Cleland CJ, Abraham WT, Linde C, et al. An individual patient meta-analysis of five randomized trials assessing the effects of cardiac resynchronization therapy on morbidity and mortality in patients with symptomatic heart failure. Eur Heart J 2013; 34: 3547-56.

2. Rickard J, Kumbhani DJ, Popovic Z, et al. Characterization of super-response to cardiac resynchronization therapy. Heart Rhythm 2010; 7: 885-9.

3. Ponikowski P, Voors AA, Anker SD, et al. 2016 ESC Guidelines for the diagnosis and treatment of acute and chronic heart failure: The Task Force for the diagnosis and treatment of acute and chronic heart failure of the European Society of Cardiology (ESC) developed with the special contribution of the Heart Failure Association (HFA) of the ESC. Eur Heart J 2016; 37: 2129-200.

4. Guédon-Moreau L, Lacroix D, Sadoul N, et al. A randomized study of remote follow-up of implantable cardioverter defibrillators: safety and efficacy report of the ECOST trial. Eur Heart J 2013; 34: 605-14.

5. Ammannaya G. Implantable cardioverter defibrillators the past, present and future. Arch Med Sci Atheroscler Dis 2020; 5: 163-70.

6. Sack S, Wende CM, Nägele $H$, et al. Potential value of automated daily screening of cardiac resynchronization therapy defibrillator diagnostics for prediction of major cardiovascular events: results from Home-CARE 
(Home Monitoring in Cardiac Resynchronization Therapy) study. Eur J Heart Fail 2011; 13: 1019-27.

7. Hernández-Madrid A, Lewalter T, Proclemer A, et al. Remote monitoring of cardiac implantable electronic devices in Europe: results of the European Heart Rhythm Association survey. Europace 2014; 16: 129-32.

8. Wetze U, Geller JC, Kautzner J, et al. Remote follow-up for ICD-therapy in patients meeting MADIT II criteria the REFORM trial. Eur Heart J 2009; 6 (5 Suppl 1): 418.

9. Zanaboni P, Landolina M, Marzegalli M, et al. Cost-utility analysis of the EVOLVO study on remote monitoring for heart failure patients with implantable defibrillators: Randomized controlled trial. J Med Internet Res 2013; 15: e106.

10. Lazarus A. Remote, wireless, ambulatory monitoring of implantable pacemakers, cardioverter defibrillators, and cardiac resynchronization therapy systems: analysis of a worldwide database. Pacing Clin Electrophysiol 2007; 30 Suppl 1: S2-S12.

11. Whellan DJ, Ousdigian KT, Al-Khatib SM, et al.; PARTNERS Study Investigators. Combined heart failure device diagnostics identify patients at higher risk of subsequent heart failure hospitalizations. J Am Coll Cardiol 2010; 55: 1803-10.

12. Tajstra M, Sokal A, Gadula-Gacek E, et al. Remote Supervision to Decrease Hospitalization Rate (RESULT) study in patients with implanted cardioverter-defibrillator. Europace 2020; 22: 769-76.

13. Hindricks G, Taborsky M, Glikson M, et al. Implant-based multiparameter telemonitoring of patients with heart failure (IN-TIME): a randomized controlled trial. Lancet 2014; 384: 583-90.

14. Boriani G, Quesada A, Ricci RP, et al. Effects of remote monitoring on clinical outcomes and use of healthcare resources in heart failure patients with biventricular defibrillators: results of the MORE-CARE multicenter randomized controlled trial. Eur J Heart Fail 2017; 19 416-25.

15. Klersy C, Boriani G, De Silvestri A, et al. Effect of telemonitoring of cardiac implantable electronic devices on healthcare utilization: a meta-analysis of randomized controlled trials in patients with heart failure. Eur J Heart Fail 2016; 18: 195-204.

16. Parthiban N, Esterman A, Mahajan R, et al. Remote monitoring of implantable cardioverter-defibrillators: a systematic review and meta-analysis of clinical outcomes. J Am Coll Cardiol 2015; 65: 2591-600.

17. Varma N, Epstein AE, Schweikert R, Michalski J, Love CJ. Role of automatic wireless remote monitoring immediately following ICD Implant: the Lumos-T Reduces Routine Office Device Follow-Up Study (TRUST) trial. J Cardiovasc Electrophysiol 2016; 27: 321-6.

18. Eisner $\mathrm{CH}$, Sommer P, Piorkowski C, et al. A prospective multicenter comparison trial of home monitoring against regular follow-up in MADIT II patients: additional visits and cost impact. Comput Cardiol 2006; 33: 241-4.

19. Varma N, Michalski J, Epstein AE, Schweikert R. Automatic remote monitoring of implantable cardioverter-defibrillator lead and generator performance the lumos-T safely reduces routine office device follow-up (TRUST) Trial. Circ Arrhythmia Electrophysiol 2010; 3: 428-36.

20. Guiédon-Moreau L, Lacroix D, Sadoul N, et al. Costs of remote monitoring vs. ambulatory follow-ups of implanted cardioverter defibrillators in the randomized ECOST study. Europace 2014; 16: 1181-8.
21. Burri H, Sticherling C, Wright D, et al. Cost-consequence analysis of daily continuous remote monitoring of implantable cardiac defibrillator and resynchronization devices in the UK. Europace 2013; 15: 1601-8.

22. Boriani G, Auricchio A, Klersy C, et al. Healthcare personnel resource burden related to in-clinic follow-up of cardiovascular implantable electronic devices: a European Heart Rhythm Association and Eucomed joint survey. Europace 2011; 13: 1166-73.

23. Vamos M, Nyolczas N, Bari Zs, et al. Refined heart failure detection algorithm for improved clinical reliability of OptiVol alerts in CRT-D recipients. Cardiol J 2018; 25: 236-44.

24. Hindricks $G$, Varma N, Kacet $S$, et al. Daily remote monitoring of implantable cardioverter-defibrillators: in sights from the pooled patient - level data from three randomized controlled trials (IN-TIME, ECOST, TRUST). Eur Heart J 2017; 38: 1749-55.

25. Sasaki S, Ishida Y, Kinjo T, et al. Telediagnosis of heart failure with continuous intrathoracic impedance monitoring by Medtronic CareLink Network: importance of the elevation pattern of OptiVol Fluid Index. J Arrhythmia 2013; 29: 347-52.

26. Bohm M, Drexler H, Oswald $H$, et al. Fluid status telemedicine alerts for heart failure: a randomized controlled trial. Eur Heart J 2016; 37: 3154-63.

27. Luthje L, Vollmann D, Seegers J, et al. A randomized study of remote monitoring and fluid monitoring for the management of patients with implanted cardiac arrhythmia devices. Europace 2015; 17: 1276-81.

28. Conraads VM, Tavazzi L, Santitini M, et al. Sensitivity, and positive predictive value of implantable intrathoracic impedance monitoring as predictor of heart failure hospitalizations: the SENSE-HF trial. Eur Heart J 2011; 32: 2266-73.

29. Heist EK, Herre JM, Binkley PF, et al. Analysis of different device-based intrathoracic impedance vectors for detection of heart failure status events (from the Detect Fluid Early from Intrathoracic Impedance Monitoring study). Am J Cardiol 2014; 114: 1249-56.

30. Maclntyre CJ, Sapp JL, Abdelwahab A, et al. The effect of shock burden on heart failure and mortality. CJC Open 2019; 1: 161-7.

31. Poole JE, Johnson GW, Hellkamp AS, et al. Prognostic importance of defibrillator shocks in patients with heart failure. N Engl J Med 2008; 359: 1009-17.

32. Fox K, Ford I, Steg PhG, et al. Relationship between ivabradine treatment and cardiovascular outcomes in patients with stable coronary artery disese and left ventricular systolic dysfunction with limiting angina: a subgroup anlysis of randomized controlled BEAUTIFUL trial. Eur Heart J 2009; 30: 2337-45.

33. Böhm M, Swedberg K, Komajda M, et al. Herat rate as risk factor in chronic heart failure (SHIFT): the association between heart rate and outcomes in a randomized placebo-controlled trial. Lancet 2010; 376: 866-94.

34. Adamson PB, Smith AL, Abraham WT, et al. Continuous autonomic assessment in patients with symptomatic heart failure: prognostic value of heart rate variability measured by an implanted cardiac resynchronization device. Circulation 2004; 110: 2389-94.

35. Hayes DL, Boehmer JP, Day JD, et al. Cardiac resynchronization therapy and the relationship of percent biventricular pacing to symptoms and survival. Heart Rhythm 2011; 8: 1469-75.

36. Landolina M, Perego GB, Lunati M, et al. Remote monitoring reduces healthcare use and improves quality of 
care in heart failure patients with implantable defibrillators: the evolution of management strategies of heart failure patients with implantable defibrillators (EVOLVO) study. Circulation 2012; 125: 2985-92.

37. Polikandrioti M, Koutelekos I, Panoutsopoulos G, et al. Hospitalized patients with heart failure: the impact of anxiety, fatigue, and therapy adherence on quality of life. Arch Med Sci Atheroscler Dis 2019; 4: 268-79.

38. Abraham WT, Fiuzat M, Psotka MA, O'Connor CM, et al. Heart failure collaborator statement on remote monitoring and social distancing in the landscape of the COVID-19. JACC Heart Fail 2020; 8: 692-4.

39. Cleland GF, Clark RA, Pellicori P, Inglis SC. Caring for people with heart failure and many other medical problems through and beyond the COVID-19 pandemic: the ad vantages of universal access to home telemonitoring Eur J Heart Fail 2020; 22: 995-8. 